\title{
Exponentially Fitted Fifth-Order Two-Step Peer Explicit Methods
}

\author{
M. Calvo*, J. I. Montijano*, L. Rández* and M. Van Daele ${ }^{\dagger}$ \\ *Instituto Universitario de Matemáticas y Aplicaciones. \\ Departamento de Matemática Aplicada. \\ Facultad de Ciencias. Universidad de Zaragoza. \\ Pedro Cerbuna 12, 50009 Zaragoza \\ $\dagger$ Vakgroep Toegepaste Wiskunde en Informatica. \\ Universiteit Gent, Krijgslaan 281-59. \\ B9000 Gent, Belgium.
}

\begin{abstract}
.
The so called peer methods for the numerical solution of Initial Value Problems (IVP) in ordinary differential systems were introduced by $\mathrm{R}$. Weiner et al $[6,7,11,12,13]$ for solving different types of problems either in sequential or parallel computers. In this work, we study exponentially fitted three-stage peer schemes that are able to fit functional spaces with dimension six. Finally, some numerical experiments are presented to show the behaviour of the new peer schemes for some periodic problems.
\end{abstract}

Keywords: Exponential fitting, Peer methods, oscillatory initial value problems

PACS: $02.60 . \mathrm{Lj}$

\section{INTRODUCTION}

We consider the numerical solution of IVPs for first order differential systems

$$
\frac{\mathrm{d}}{\mathrm{d} t} y(t)=f(t, y(t)), \quad t \in\left[t_{0}, t_{0}+T\right], \quad y\left(t_{0}\right)=y_{0} \in \mathbf{R}^{m},
$$

where for simplicity $f(t, y)$ is assumed to be sufficiently smooth, so that the IVP (1) has a unique solution. Furthermore, some knowledge of the behaviour of their unique global solution is known in advance. In the case that the solution of (1) has an oscillatory behaviour and further we know an estimate of the frequency, some modified Runge-Kutta (RK) methods using this information, usually called trigonometrically fitted or more generally exponentially fitted methods $[3,2,4,5,9,10]$ have been proposed in the last years to improve their accuracy and efficiency over standard RK methods.

For explicit RK methods the stage order is limited to one and this implies serious restrictions in the dimensionality of the fitting space. On the other hand, linear multistep methods do not have such a limitation, as shown for example in the early paper of Gautschi [3]. In this case, with $k$ steps, a method can be fitted to $k+1$ dimensional spaces.

Here, we consider explicit two-step peer methods introduced by R. Weiner, et al [6, 7] as an alternative to classical Runge-Kutta (RK) and multistep methods attempting to combine the advantages of these two classes of methods.

In particular fitted three-stage peer methods based on the previous schemes given in [1] to some fitting spaces are constructed.

Some numerical experiments are presented to show the performance of the above fitted methods for problems with oscillatory solutions. The proposed methods are compared to exponentially fitted Adams-Bashforth-Moulton methods with the same order. 


\section{FITTED TWO STEP PEER METHODS}

It will be sufficient to consider in (1) scalar equations $(m=1)$ and then a $s$-stage peer scheme can be expressed in vector form as

$$
\mathbf{Y}_{1}=A \mathbf{Y}_{0}+h B \mathbf{F}_{0}+h R \mathbf{F}_{1}
$$

where the $s$-dim vector $\mathbf{Y}_{j}=\left\{Y_{j, l}\right\}$ includes approximations to the solution of (1) at $t_{j}+c_{l} h, l=1, \ldots, s$ and

$$
\begin{aligned}
\mathbf{Y}_{k} & =\left(Y_{k, 1}, \ldots, Y_{k, s}\right)^{T} \in \mathbf{R}^{s} \\
\mathbf{e} & =(1, \ldots, 1)^{T} \in \mathbf{R}^{s}, \\
\mathbf{c} & =\left(c_{1}, \ldots, c_{s}\right)^{T} \in \mathbf{R}^{s}, \\
\mathbf{F}_{k} & =f\left(t_{k} \mathbf{e}+h \mathbf{c}, \mathbf{Y}_{k}\right)=\left(f\left(t_{k}+h c_{j}, Y_{k, j}\right)\right)_{j=1}^{s} \in \mathbf{R}^{s},
\end{aligned}
$$

where $A \in \mathbf{R}^{s \times s}$ and $B \in \mathbf{R}^{s \times s}$ full matrices and $R \in \mathbf{R}^{s \times s}$ strictly lower triangular are the free parameters that define the method and the set of admissible fixed nodes $c_{j}, j=1, \ldots, s$ satisfies that $\left|c_{i}-c_{j}\right| \neq 0,1$ for all $i \neq j$.

It is worth to note that (2) is a multivalued method and 0 -stability requires that the matrix $A$ satisfies the root's condition. In this work we consider only methods with the requirement that the eigenvalues $\lambda_{j}(A)$ of $A$,

$$
\lambda_{1}(A)=1, \quad \lambda_{j}(A)=0, \quad j=2, \ldots, s .
$$

Furthermore, following the ideas of [1], in order to simplify the derivation of the fitting methods and to get methods with high stage order, we will take $A$ with the form

$$
A=P^{-1} \widehat{A} P
$$

with $P=\left(p_{i j}\right) \in \mathbf{R}^{s \times s}$ a lower triangular matrix with ones at the diagonal, and $\widehat{A}=\left(\hat{a}_{i j}\right) \in \mathbf{R}^{s \times s}$ upper triangular whose $\operatorname{diagonal}$ is $\operatorname{diag}(\widehat{A})=(1,0, \ldots, 0)$, that clearly satisfy (4).

To study the local discretization error, we associate to (2) the linear $s$-dimensional vector valued operator $\mathscr{L}[\varphi ; h]$ defined on sufficiently smooth scalar real or complex functions $\varphi$ by

$$
\mathscr{L}[\varphi ; h](t)=\varphi((t+h) \mathbf{e}+h \mathbf{c})-A \varphi(t \mathbf{e}+h \mathbf{c})-h B \dot{\varphi}(t \mathbf{e}+h \mathbf{c})-h R \dot{\varphi}((t+h) \mathbf{e}+h \mathbf{c}) .
$$

For our class of strongly 0 -stable peer methods where $A$ is given by (5) we have

$$
\mathscr{L}[\varphi ; h]\left(t_{0}\right)=P^{-1} \widehat{\mathscr{L}}[\varphi ; h]\left(t_{0}\right),
$$

with

$$
\widehat{\mathscr{L}}[\varphi ; h]\left(t_{0}\right)=\mathbf{Z}(t+h)-\widehat{A} \mathbf{Z}(t)-h \widehat{B} \dot{\mathbf{Z}}(t)-h \widehat{R} \dot{\mathbf{Z}}(t+h),
$$

and $\mathbf{Z}(t)=P \varphi(t \mathbf{e}+h \mathbf{c})$. In view of (7) the method (2) is fitted to the functional space $\mathscr{F}_{q}$ at $t_{0}$ with step size $h$ iff

$$
\widehat{\mathscr{L}}[\varphi ; h]\left(t_{0}\right)=0, \quad \forall \varphi \in \mathscr{F}_{q} .
$$

We can state the next important result:

Theorem.- Suppose that for a given set of admissible fixed nodes and constant matrix $P$ the standard two-step peer method (2), (5) with $s$ stages has a unique solution with stage order $2 s-1$, then:

1. For any linear space $\mathscr{F}_{2 s-1}=\left\langle 1, \varphi_{1}(t), \ldots, \varphi_{2 s-1}(t)\right\rangle$ there exists a unique $s$-stage two step peer method fitted to this space for $h$ sufficiently small. This peer method has the same nodes and $P$-matrix as the standard method and the coefficients

$$
\widehat{A}_{\mathscr{F}}=\widehat{A}\left(t_{0}, h\right), \quad \widehat{B}_{\mathscr{F}}=\widehat{B}\left(t_{0}, h\right), \quad \widehat{R}_{\mathscr{F}}=\widehat{R}\left(t_{0}, h\right),
$$

may depend (apart from the fitting space) on $t_{0}$ and $h$.

2. If $\mathscr{F}_{2 s-1}$ is a separable basis the coefficients are independent of $t_{0}$. 


\section{THREE-STAGE EXPONENTIALLY FITTED PEER METHODS}

In this section, we consider exponential fitted variants for the fifth order peer scheme deduced in [1], which are fitted to the particular spaces

- $\mathscr{F}_{5}=\left\langle 1, t, t^{2}, t^{3}, t^{4}, t^{5}\right\rangle$

- $\mathscr{F}_{5}(\omega)=\left\langle 1, t, t^{2}, t^{3}, \sin (\omega t), \cos (\omega t)\right\rangle$

- $\mathscr{F}_{5}\left(\omega_{1}, \omega_{2}\right)=\left\langle 1, t, \sin \left(\omega_{1} t\right), \cos \left(\omega_{1} t\right), \sin \left(\omega_{2} t\right), \cos \left(\omega_{2} t\right)\right\rangle$

where $\omega, \omega_{1}, \omega_{2}$ are fixed positive parameters.

Due to the linear, explicit, multistep nature of each stage of the PEER schemes, we use predictor-corrector pairs of linear multistep methods for comparison. We choose the classical four-step Adams-Bashforth-Moulton pair of order 5 (denoted by AM5), the fitted versions of the previous scheme to $\mathscr{F}_{5}(\omega)$ (denoted by AM5 $\left.(\omega)\right)$, to $\mathscr{F}_{5}\left(\omega_{1}, \omega_{2}\right)($ denoted by $\left.\operatorname{AM} 5\left(\omega_{1}, \omega_{2}\right)\right)$. The fitted multistep methods have been obtained following the ideas in [3].

We have implemented the AM5 schemes in the mode PECE, that requires only 2 function evaluations per step. For the PEER3 methods, 3 function evaluations per step are needed, so in order to be able to compare the computational cost (total number of function evaluations), we have applied PEER3 methods with stepsizes $50 \%$ larger than those of the AM5 methods.

The criterion used in the numerical comparisons is the maximum global error in the solution over the whole integration interval. All computations were carried out in quadruple precision arithmetic (32 significant digits of accuracy) on a PC computer running PYTHON.

\section{Problem}

We consider the two-dimensional semi-linear oscillatory second-order IVP

$$
q^{\prime \prime}(t)=-\frac{1}{2}\left(\begin{array}{ll}
w^{2}+k^{2}+1 & w^{2}-k^{2}-1 \\
w^{2}-k^{2}-1 & w^{2}+k^{2}+1
\end{array}\right) q(t)+\frac{k^{2}}{2}\left(q_{1}(t)-q_{2}(t)\right)^{3}\left(\begin{array}{c}
1 \\
-1
\end{array}\right)
$$

with initial conditions

$$
q(0)=\left(\begin{array}{c}
-1 / 2 \\
-1 / 2
\end{array}\right), \quad q^{\prime}(0)=\frac{1}{2}\left(\begin{array}{c}
-\sqrt{2}-w \\
\sqrt{2}-w
\end{array}\right)
$$

and the parameters $w>0,0 \leq k<1$. This problem represents a simple model consisting of two point masses connected by a soft nonlinear spring and a stiff linear one, and its exact periodic solution can be written in terms of the Jacobi's elliptic functions as

$$
q(t)=\frac{\sqrt{2}}{2}\left(\begin{array}{c}
\cos (\pi / 4+w t)-\operatorname{sn}(t, k) \\
\cos (\pi / 4+w t)+\operatorname{sn}(t, k)
\end{array}\right)
$$

In our numerical experiments we have taken $w=2, k=0.05, t_{\text {end }}=100$, and $h=1 /\left(5 \times 2^{i}\right), i=1, \ldots, 5$ and the numerical results are presented in Figure 1.

In this test problem, the efficiency of the peer schemes considered is slighty superior to the corresponding multistep methods. Also, the methods fitted with two frequencies $\omega_{1}=2, \omega_{2}=1$ perform much better than the corresponding schemes fitted only to one frequency.

\section{REFERENCES}

1. M. Calvo, J.I. Montijano, L. Rández and M.Van Daele, On the derivation of explicit two step peer methods, Appl. Numer. Math. 61 (2011), n. 4, 395-409.

2. J.M. Franco, Runge-Kutta methods adapted to the numerical integration of oscillatory problems, Appl. Numer. Math. 50 (2004) 427-443.

3. W. Gautschi, Numerical integration of ordinary differential equations based on trigonometric polynomials, Numer. Math., 3 , (1961) 381-397.

4. L.Gr. Ixaru, and G. Vanden Berghe, Exponential Fitting, Mathematics and Its Applications vol. 568, Kluwer, Dordrecht, 2004

5. B. Paternoster, Runge-Kutta (-Nyström) methods for ODEs with periodic solutions based on trigonometric polynomials. Applied Numerical Matehmatics 28, 401-412 (1998).

6. B.A. Schmitt, R. Weiner, Parallel two-step W-methods with peer variables, SIAM J. Numer. Anal. 42 (2004), no. 1, $265-282$.

7. B.A. Schmitt, R. Weiner, K. Erdmann, Implicit parallel peer methods for stiff initial value problems, Appl. Numer. Math. 53 (2005), no. 2-4, 457-470. 


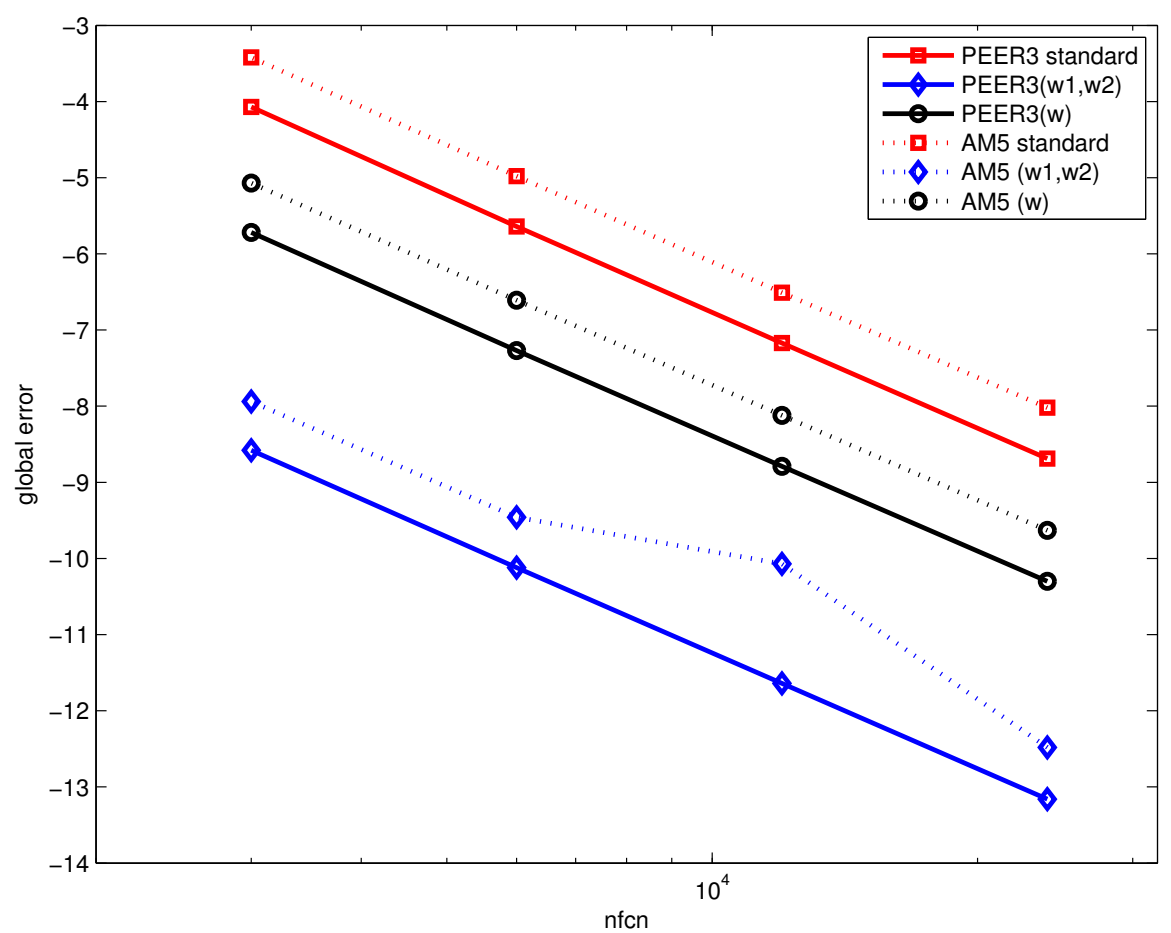

FIGURE 1. Efficiency plot of fifth-order schemes for the two-dimensional semi-linear oscillatory problem with $\omega_{1}=\omega=2$, $\omega_{2}=1, k=0.05$

8. B.A. Schmitt, R. Weiner, S. Jebens, Parameter optimization for explicit parallel peer two-step methods. Appl. Numer. Math. 59, 769-782 (2009).

9. G. Vanden Berghe, H. De Meyer, M. Van Daele and T. Van Hecke, Exponentially-fitted explicit Runge-Kutta methods. Comput. Phys. Comm. 123 7-15 (1999).

10. G. Vanden Berghe and M. Van Daele, Trigonometric polynomial or exponential fitting approach?. Journal of Computational and Applied Mathematics, 233, 4, 969-979, (2009).

11. R. Weiner, B.A. Schmitt, H. Podhaisky, Linearly-implicit two-step methods and their implementation in Nordsieck form, Appl. Numer. Math. 56 (2006), no. 3-4, 374-387.

12. R. Weiner, B.A. Schmitt, H. Podhaisky, S. Jebens, Superconvergent explicit two-step peer methods, Journal of Computational and Applied Mathematics, 223, 753-764 (2009).

13. R. Weiner, K. Biermann, B.A. Schmitt, H. Podhaisky, Explicit two-step peer methods, Computers and Mathematics with Applications 55, 609-619 (2008). 